\title{
Tenfold Way for Quadratic Lindbladians
}

\author{
Simon Lieu, Max McGinley®, and Nigel R. Cooper® \\ T.C.M. Group, Cavendish Laboratory, University of Cambridge, JJ Thomson Avenue, \\ Cambridge CB3 OHE, United Kingdom
}

(Received 24 August 2019; revised manuscript received 14 November 2019; published 27 January 2020)

\begin{abstract}
We uncover a topological classification applicable to open fermionic systems governed by a general class of Lindblad master equations. These "quadratic Lindbladians" can be captured by a non-Hermitian single-particle matrix which describes internal dynamics as well as system-environment coupling. We show that this matrix must belong to one of ten non-Hermitian Bernard-LeClair symmetry classes which reduce to the Altland-Zirnbauer classes in the closed limit. The Lindblad spectrum admits a topological classification, which we show results in gapless edge excitations with finite lifetimes. Unlike previous studies of purely Hamiltonian or purely dissipative evolution, these topological edge modes are unconnected to the form of the steady state. We provide one-dimensional examples where the addition of dissipators can either preserve or destroy the closed classification of a model, highlighting the sensitivity of topological properties to details of the system-environment coupling.
\end{abstract}

DOI: 10.1103/PhysRevLett.124.040401

Introduction.-Topological band theory was developed to predict and explain robust features in the electronic structure of insulators and superconductors close to their ground states $[1,2]$. While these ideas have already found fundamental applications in quantum metrology [3] and quantum computation [4], there has been a recent effort to understand the role of topology in the dynamics of many-body systems in highly nonequilibrium environments [5-12].

A growing body of literature has been dedicated to studying topological aspects of "non-Hermitian Hamiltonians," which generate nonunitary time evolution in certain dissipative classical and quantum settings [13-16]. While this versatile approach applies in various limits, it is insufficient to describe the full time evolution of a generic open quantum many-body system coupled to a bath. An open system is described by a (possibly mixed) density matrix $\rho$ which propagates irreversibly due to dissipative coupling with its environment. For suitably generic baths, $\rho$ is governed by the Liouville equation: $i \dot{\rho}=\mathcal{L}(\rho)$, where $\mathcal{L}$ is the "Lindbladian"- a non-Hermitian superoperator that acts linearly on $\rho(t)$. While calculating the complex spectrum of the Lindbladian can always be viewed as a non-Hermitian eigenvalue problem, $\mathcal{L}$ possesses an inherent structure which further constrains the topological signatures of open systems.

In this Letter, we show that there exists a robust topological classification of the full complex spectrum of the Lindbladian, $\mathcal{L}$, for the case of a Markovian bath with linear fermionic dissipators. In this case, the Lindblad spectral problem reduces to solving for the eigenvalues of a non-Hermitian quadratic Fermi operator $[17,18]$. An understanding of the symmetry properties of this operator allows us to compute the set of topologically distinct Lindblad spectra, which exhibit properties that are stable against continuous deformations. In particular, we make use of the real-line gap topological classification of Bernard-LeClair symmetry classes [19], recently uncovered by Kawabata et al. [14].

Surprisingly, we find that our classification-which applies in the presence of both dissipation and coherent internal dynamics - differs qualitatively from the two limiting cases that have previously been much studied, of purely Hamiltonian systems (Hermitian Lindbladian) [1,2] and of purely dissipative systems (anti-Hermitian Lindbladian) [20-23].

As in closed systems, the topological classification has consequences for dynamics near the system boundary. We show that a topologically nontrivial Lindbladian possesses robust edge modes whose phase-oscillation frequencies are pinned to lie in the energy gap, but which generically pick up finite lifetimes (see Fig. 1). (These edge modes will appear in spectroscopic measurements as broadened peaks within the bulk gap.) However, we find that, unlike previous classifications for purely Hamiltonian or purely dissipative systems, properties of the spectrum and steady state are completely independent: The existence of spectral edge modes implies nothing about the steady state density operator. For example, these universal topological properties of the complex excitation spectrum-which have direct physical consequences in spectroscopy-are unconnected to the classification of steady-state density matrices employed in Refs. [21,24-26]. Our work highlights the various manifestations of band topology in a very general class of exactly solvable open systems, and provides formalisms which can be applied to understand generic interacting systems in future work. 


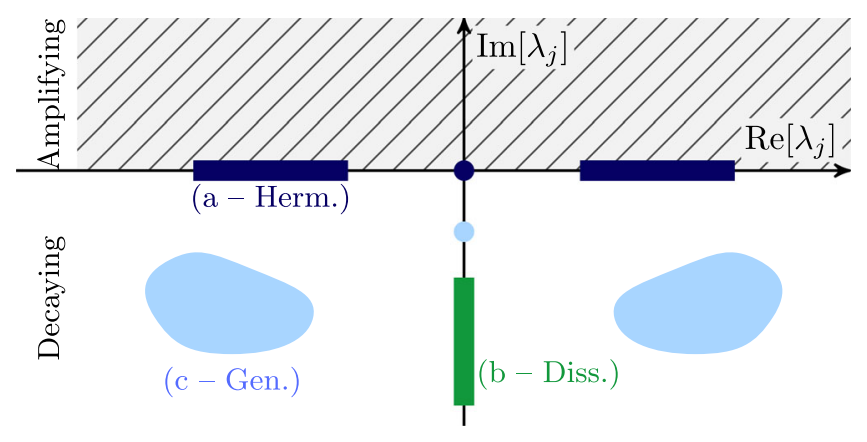

FIG. 1. Complex spectra of one-dimensional examples of [(a), dark blue] closed Hermitian systems; [(b), green] purely dissipative systems studied in Refs. [20,21,25]; and [(c), light blue] generic quadratic Lindbladians studied in this work. Hermitian systems in a topological phase possess in-gap states with zero eigenvalue (dark blue dot), however the topology of purely dissipative systems is not reflected in the Lindblad spectrum. On the other hand, a quadratic Lindbladian which is gapped in the real direction can possess robust zero-frequency edge modes (light blue dot).

Quadratic Lindbladians.-Before discussing topological edge modes in an open environment, we describe the general setup considered in this work. Our starting point is the Gorini-Kossakowski-Sudarshan-Lindblad master equation

$i \frac{d \rho}{d t}=\mathcal{L}(\rho)=[\mathcal{H}, \rho]+i \sum_{\mu}\left(2 L_{\mu} \rho L_{\mu}^{\dagger}-\left\{L_{\mu}^{\dagger} L_{\mu}, \rho\right\}\right)$

which describes nonunitary time evolution of a density matrix $\rho$ subject to unitary dynamics generated by a Hamiltonian $\mathcal{H}$ and dissipation due to operators $L_{\mu}$ which can add and/or remove particles via a Markovian environment [27]. Typically there exists a unique steady state $\rho_{\mathrm{SS}}$ satisfying $\mathcal{L} \rho_{\mathrm{SS}}=0$; all other eigenoperators have complex eigenvalues with negative imaginary part, corresponding to terms decaying in time. Note that we have multiplied the typical definition of $\mathcal{L}$ by $i$ such that the master equation resembles a non-Hermitian Schrödinger equation: Real parts of eigenvalues (called energies) indicate phase oscillation frequencies of eigenoperators, while negative imaginary parts correspond to the decay rate.

For a system of $N$ fermions, one can always solve for the spectrum $\lambda$ of the "Lindbladian" $\mathcal{L}$ by projecting onto some basis $\rho=\sum_{i, j} \rho_{i, j}\left|\phi_{i}\right\rangle\left\langle\phi_{j}\right|$, which has dimension $2^{N} \times 2^{N}=2^{2 N}$. Exact diagonalization of the resulting square matrix is numerically expensive, since the basis grows exponentially with the number of particles. However, further progress can be made if the Hamiltonian is quadratic in Fermi operators, and the dissipators are linear-such systems we refer to as quadratic Lindbladians, and are the subject of this work. In this case, Prosen $[17,18]$ showed that the spectrum of the Lindbladian can be found by diagonalizing a non-Hermitian fermionic superconductor with $2 \mathrm{~N}$ particles in Bogoliubov-de Gennes form. The factor of 2 can be understood because we assign a fermion to both "bra" and "ket" space. The number of eigenstates is again $2^{2 N}$ since each of the $2 N$ Bogoliubov quasiparticles can either be excited or not.

We briefly review this approach for $N$ complex fermions. The Hamiltonian and dissipators can be expressed in terms of $2 \mathrm{~N}$ Majorana fermions

$$
\mathcal{H}=\sum_{i, j=1}^{2 N} \alpha_{i} H_{i, j} \alpha_{j}, \quad L_{\mu}=\sum_{i=1}^{2 N} l_{\mu, i} \alpha_{i},
$$

where $H=H^{\dagger}, H=-H^{T}$. Majorana operators satisfy the anticommutation relation $\left\{\alpha_{i}, \alpha_{j}\right\}=2 \delta_{i j}$. Define a $2 N \times$ $2 N$ Hermitian matrix $M=l^{T} l^{*}$. The Lindbladian can then be represented as a superoperator acting on a doubled Hilbert space spanned by $2 N$ complex fermions $\left\{c_{j}\right\}$

$$
\mathcal{L}=2\left(\begin{array}{cc}
\mathbf{c}^{\dagger} & \mathbf{c}
\end{array}\right)\left(\begin{array}{cc}
-Z^{T} & Y \\
0 & Z
\end{array}\right)\left(\begin{array}{c}
\mathbf{c} \\
\mathbf{c}^{\dagger}
\end{array}\right)
$$

where $\mathbf{c}=\left(c_{1}, \ldots, c_{2 N}\right), Y=2 \operatorname{Im}[M], Z=H+i \operatorname{Re}[M]$. The $c$ superoperators explicitly act on the density matrix via $c_{j}^{\dagger} \rho=\left[\alpha_{j} \rho+\left(\mathcal{P}^{F} \rho\right) \alpha_{j}\right] / 2$ and $c_{j} \rho=\left[\alpha_{j} \rho-\left(\mathcal{P}^{F} \rho\right) \alpha_{j}\right] / 2$, where $\mathcal{P}^{F}$ is the fermion parity superoperator [28]. Because of this upper triangular form Eq. (3), one can now diagonalize the Lindbladian in terms of $2 \mathrm{~N}$ quasiparticles

$$
\mathcal{L}=4 \sum_{j=1}^{2 N} \lambda_{j} \bar{\beta}_{j}^{\dagger} \beta_{j}
$$

where $\lambda_{j}$ are the eigenvalues of the matrix $-Z$. Quasiparticles obey generalized fermionic statistics: $\left\{\bar{\beta}_{i}^{\dagger}, \beta_{j}\right\}=\delta_{i, j}$, $\left\{\bar{\beta}_{i}^{\dagger}, \bar{\beta}_{j}^{\dagger}\right\}=\left\{\beta_{i}, \beta_{j}\right\}=0$. In the doubled Hilbert space, the steady state is represented as a $2^{2 N}$-dimensional vector that is annihilated by all quasiparticles: $\beta_{i} \rho_{\mathrm{SS}}=0$. The states $\bar{\beta}_{i}^{\dagger} \rho_{\mathrm{SS}}$ represent eigenoperators of $\mathcal{L}$, propagating with complex energy $4 \lambda_{i}$.

The single-particle Lindblad spectrum $\{\lambda\}$ satisfies two generic conditions: (1) $\operatorname{Im}\left[\lambda_{i}\right] \leq 0$, since elements of the density matrix can only decay (not amplify) as a function of time, and (2) eigenvalues must come in anti-complexconjugate pairs $\{\lambda\}=\left\{-\lambda^{*}\right\}$ where the brackets indicate the set of spectral eigenvalues; this ensures Hermiticity of the density matrix at all times.

Non-Hermitian tenfold way.-In what follows, we will be interested in studying the robust features of the complex Lindblad spectrum associated with a topological insulator or superconductor in the presence of general linear fermionic dissipation. We begin by addressing the symmetries of the matrix whose eigenvalues determine the spectrum of quadratic Lindbladians. From Eq. (3), the upper triangular 


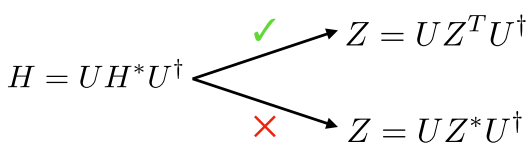

FIG. 2. Hermitian time-reversal symmetry (left) must be implemented using transposition rather than conjugation once non-Hermitian dissipative terms are included (right). $(Z=H+i \operatorname{Re}[M])$

structure of the matrix implies that the spectrum does not depend on $Y$, and hence it is fully determined from the eigenvalues of the $2 N$-dimensional square matrix $Z=H+i \operatorname{Re}[M]$.

The Hamiltonian of noninteracting fermions can be sorted into one of ten Altland-Zirnbauer [29] symmetry classes based the presence or absence of the following three symmetries:

$$
\begin{array}{clr}
\text { TRS: } & H=U_{T} H^{*} U_{T}^{\dagger}, & U_{T} U_{T}^{*}= \pm \mathbb{I} \\
\text { PHS : } & H=-U_{C} H^{*} U_{C}^{\dagger}, & U_{C} U_{C}^{*}= \pm \mathbb{I} \\
\text { chiral: } & H=-U_{S} H U_{S}^{\dagger}, & U_{S}^{2}=\mathbb{I},
\end{array}
$$

where the matrices $U_{T, C, S}$ are all unitary. Physically, these stem from time-reversal, particle-hole, and chiral (sublattice) symmetry, respectively. Our use of Majorana fermions ensures that Eq. (5b) is automatically satisfied with $U_{C}=\mathbb{I}$; however, if charge is conserved then one can decouple particle and hole sectors, each of which separately does not respect PHS. A topological classification of noninteracting models based on these ten classes is called the tenfold way [30,31], and describes symmetry-protected topological phases of free fermions.

We now ask whether $Z$ can inherit these symmetries once dissipators are introduced, i.e., $M \neq 0, Z \neq Z^{\dagger}$. If TRS is imposed on $Z$ in the form Eq. (5), i.e., $Z=U_{T} Z^{*} U_{T}^{\dagger}$, then we will find that a damping mode with eigenvalue $\lambda$ must be paired with a mode of eigenvalue $\lambda^{*}$-this has the same frequency $\operatorname{Re}[\lambda]$ but a negative damping rate $\operatorname{Im}[\lambda]$, and is thus unphysical. (See Fig. 2.) Similarly, PHS cannot be represented via an expression of the form $Z=-U_{C} Z^{T} U_{C}^{\dagger}$, since this would ensure that eigenvalues come in positivenegative pairs: $\{\lambda\}=\{-\lambda\}$. Indeed $Z$ cannot respect any symmetry which associates a decaying mode with an amplifying one. We find a unique way to extend the Hamiltonian symmetries, Eq. (5), to Lindbladian symmetries which does not suffer from this problem, namely,

$$
\begin{array}{ccc}
\text { TRS: } & Z=U_{T} Z^{T} U_{T}^{\dagger}, & U_{T} U_{T}^{*}= \pm \mathbb{I} \\
\text { PHS: } & Z=-U_{C} Z^{*} U_{C}^{\dagger}, & U_{C} U_{C}^{*}= \pm \mathbb{I} \\
\text { PAH: } & Z=-U_{S} Z^{\dagger} U_{S}^{\dagger}, & U_{S}^{2}=\mathbb{I} .
\end{array}
$$

Different combinations of these symmetries generate ten Lindbladian symmetry classes which reduce to the AltlandZirnbauer classes in the absence of dissipation. While the non-Hermitian Bernard-LeClair symmetries generate a much larger number of unique classes compared to their Hermitian counterparts [19], the inherent structure of quadratic Lindbladians ensures that the spectral matrix $Z$ must belong to one of the ten classes defined above. Although the new form of time-reversal symmetry appears unusual, we show in the Supplemental Material [32] that this symmetry arises naturally when the microscopic Hamiltonian of the system and environment as a whole respect the Hermitian TRS, Eq. (5) (even though the system alone propagates irreversibly). Note also that pseudo-antiHermiticity (PAH) generalizes chiral symmetry, i.e., it is guaranteed if a model has TRS and PHS.

Recent studies have used Bernard-LeClair symmetries to construct a topological classification for non-Hermitian models [14]. In this context, there exist different choices for defining a spectral gap-some range of energy within which no bulk eigenvalues are present. The positivity condition $\operatorname{Im}\left[\lambda_{i}\right] \leq 0$ again puts constraints on these possibilities. If one chooses a point gap at the origin $\left(\lambda_{i} \neq 0\right)$, or an imaginary line gap $\left(\operatorname{Im}\left[\lambda_{i}\right] \neq 0\right)$, then the eigenvalues of $Z$ can be continuously deformed to a single point without crossing these gaps, and so an analysis under these conditions will not identify any robust spectral properties. However, one can choose a real line gap condition $\operatorname{Re}\left[\lambda_{i}\right] \neq 0$, i.e., we insist that all bulk modes have a finite oscillation frequency [Fig. 1(c)]. Note that this is in stark contrast to the pure-dissipation case $[20,21,25]$.

According to Ref. [14], the classification table for the ten Bernard-LeClair classes which stem from Eqs. (6) under a real line gap is the same as that for the conventional tenfold way, once the non-Hermitian symmetry classes are associated with their corresponding Hermitian counterparts. The relevant bulk topological indices can be calculated for all the negative-frequency bands, and if their sum is nonzero then we expect in-gap states to appear at the system boundary, just as in Hermitian band theory. Since the gap is chosen along the imaginary axis, an edge mode of the Lindbladian will be pinned to zero frequency, but generically will have a finite damping rate, since the classification is only sensitive to $\operatorname{Re}\left[\lambda_{i}\right]$.

An intuitive picture is formed if one takes a topologically nontrivial system and gradually turns on dissipators without closing the frequency gap. If this procedure is carried out while at all times respecting the symmetries Eq. (6), then the topological classification of the new open system is identical to its closed precursor. Our results show that the gapless edge modes of the Hermitian system will remain constrained to lie in the middle of the gap, and acquire a finite lifetime. Similarly, as was found for the SuSchrieffer-Heeger (SSH) chain in Ref. [37], topological invariants can be defined for the spectrum of the open 
system such that they are equal to those for the closed system.

Independence of steady-state properties.-In isolated systems, the topological properties of the ground state are reflected in the spectrum of the Hamiltonian. In open systems, the analogous state to consider is the nonequilibrium steady state $\mathcal{L}\left(\rho_{\mathrm{SS}}\right)=0$. Although $\rho_{\mathrm{SS}}$ is generically not a pure state, one can still discuss its topological properties by using appropriate invariants for density matrices [25]. Studies of systems with pure dissipation $(\mathcal{H}=0)$ have shown that an alternative tenfold way for open systems arises based on these properties [20,21,24,26]. One might expect that our spectral analysis reflects these steady state properties, in parallel with closed systems.

However, we find that the spectral and steady state topological properties of quadratic Lindbladians are independent. We prove this by showing that for any Lindbladian with a nontrivial steady state, there exists another Lindbladian with the same symmetries and spectrum, but with a trivial steady state. This auxiliary system has the same Hamiltonian, but the (generally complex) dissipators $\ell_{\mu, i}$ are replaced by real values $\tilde{\ell}_{\mu, i}$ which satisfy $\tilde{\ell}^{T} \tilde{\ell}=M \equiv \operatorname{Re}\left(\ell^{T} \ell^{*}\right)$. Because the matrix $Z$ depends only on $M$ and $H$, the spectrum is unaffected. However, one finds that $\rho_{\mathrm{SS}} \propto \mathbb{I}$, and is thus always a structureless "trivial" steady state. In the Supplemental Material [32], we show that a valid $\tilde{\ell}_{\mu, i}$ always exists and is sufficiently local such that one can define a continuous path of Lindbladians that leaves the spectrum invariant (e.g., without closing the gap in real frequency) yet connects the physical system to this auxiliary system with a trivial steady state. Hence, the form of the spectrum is unconnected to the form of the steady state.

Having uncovered the general symmetry-based topological classification of quadratic Lindbladians, we now illustrate its relevant features in the context of an example system.

Dissipative Kitaev chain. - We consider the Kitaev chain [38] in the presence of local, linear dissipators. The unitary evolution is generated via the Hamiltonian

$$
\mathcal{H}_{\mathrm{Kit}}=i \mu \sum_{j=1}^{N} \alpha_{j, A} \alpha_{j, B}+i \Delta \sum_{j=1}^{N-1} \alpha_{j, B} \alpha_{j+1, A}
$$

where $\alpha_{j, A / B}$ represent the two types of Majorana fermions on lattice site $j$ of $N$, and $\mu, \Delta \in \mathbb{R}$. We also consider $N-1$ dissipators which connect nearest-neighbor sites: $L_{j}=$ $\gamma\left(\alpha_{j, A}+i \alpha_{j+1, B}\right)$. A variant of this model has been studied previously [28]; however, we shall emphasize the importance of the non-Hermitian Bernard-LeClair symmetries which are responsible for the protection of gapless edge modes.

The Kitaev chain Hamiltonian falls into class BDI, which has a $\mathbb{Z}$ classification in $1 D$. In a Majorana basis, the first-quantized (matrix) Hamiltonian obeys the symmetries $H=-H^{*}, H=\tau_{z} H^{*} \tau_{z}, H=-\tau_{z} H \tau_{z}$ where

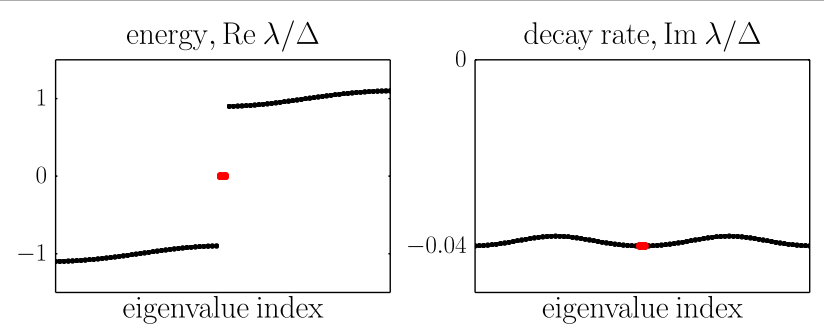

FIG. 3. Lindblad spectrum for the Kitaev chain with linear, nearest-neighbor dissipators, $\mu / \Delta=0.1, \gamma^{2} / \Delta=0.04$. A single edge mode exists on each side of the chain (red dots), and is symmetry protected to obey $\operatorname{Re}\left[\lambda_{\text {edge }}\right]=0$. Majorana edge modes of the closed system can couple to fermionic dissipators and hence acquire a finite lifetime $\operatorname{Im}\left[\lambda_{\text {edge }}\right]<0$.

$\tau_{z}=\mathbb{I}_{N} \otimes \sigma_{z}$, and $\sigma_{z}$ is the Pauli matrix which acts on the Majorana sublattice index. If we turn on the dissipator strength $\gamma \neq 0$, then the dynamics of the open system is determined from the Lindblad spectrum, found explicitly by diagonalizing $Z$. $Z$ inherits the following symmetries: $Z=-Z^{*}, Z=\tau_{z} Z^{T} \tau_{z}, Z=-\tau_{z} Z^{\dagger} \tau_{z}$. Indeed we find that such dissipators will keep the model in the same symmetry class, and we expect the edge modes to obey $\operatorname{Re}\left[\lambda_{\text {edge }}\right]=0$. For spinless fermions, any dissipator which can be written in the form $L_{\mu}=e^{i \phi_{\mu}} \sum_{j}\left(\gamma_{\mu, j} \alpha_{j, A}+i \bar{\gamma}_{\mu, j} \alpha_{j, B}\right)$, for $\phi_{\mu}, \gamma_{\mu, j}$, $\bar{\gamma}_{\mu, j} \in \mathbb{R}$ will preserve the TRS condition Eq. (6).

The spectrum is calculated numerically, and plotted in Fig. 3. We notice that indeed edge modes are constrained to obey $\operatorname{Re}\left[\lambda_{\text {edge }}\right]=0$, while the imaginary part of their energy becomes negative. Mathematically, this is due to pseudoanti-Hermiticity: $Z=-\tau_{z} Z^{\dagger} \tau_{z}$ which implies $\lambda_{\text {edge }}=$ $-\lambda_{\text {edge }}^{*} \Rightarrow \operatorname{Re}\left[\lambda_{\text {edge }}\right]=0, \operatorname{Im}\left[\lambda_{\text {edge }}\right] \neq 0[39]$. We can also understand this behavior physically: The linear fermionic dissipators break fermion parity conservation of the closed Kitaev chain, hence Majorana modes at a given edge can couple to the environment and will acquire a finite lifetime (called quasiparticle poisoning) [28,40,41]. If, instead, dissipators obeyed fermion parity then we would expect the steady state to retain its twofold degeneracy due to decoupled parity sectors. (This type of dissipation falls outside the scope of quadratic Lindbladians.) Coupling to dissipators cannot, however, perturb the frequency of edge mode phase oscillations, since we have demonstrated that symmetries protect these zero-frequency eigenoperators of the Lindbladian.

The spectrum of the Lindbladian can be inferred from single-particle Green's functions in the frequency domain, i.e., the Fourier transform of $\left\langle\alpha_{i}(t) \alpha_{j}(0)\right\rangle$. A particular eigenvalue $\lambda$ will give rise to a spectroscopic peak centred on $\operatorname{Re}[\lambda]$ with a characteristic width $\operatorname{Im}[\lambda]$. In experiment, these can be determined from linear response functions (see, e.g., Refs. [42,43]). For example, the zero-bias tunneling peak characteristic of Majorana modes in topological superconductors should remain centered at zero energy, but acquire a finite width. 
In the Supplemental Material [32], we discuss a different example (an open SSH chain) where the relevant symmetries can be either preserved or violated by the dissipators [whereas the PHS, Eq. (6b), intrinsic to superconducing systems cannot be broken].

Outlook.-An immediate question is whether gapless edge modes can exist in the imaginary spectrum, which would lead to robustly nonunique steady-state density matrices. While certain studies [20,21] have achieved this via "topology by dissipation" where Hamiltonian dynamics is fully switched off, such edge modes generically acquire a lifetime once Hamiltonian terms are added back, implying that this effect is fragile against such local perturbations. The existence of such a protected in-gap state for free fermions would require bands which amplify and bands which decay, such that the edge mode connects the two bulk bands. This scenario is forbidden, since the imaginary Lindblad spectrum is constrained to be nonpositive.

While we have limited our discussion to the case of "quadratic Lindbladians," we expect the topological edge modes described in this work to survive beyond this limit as non-Hermitian analogues of interacting symmetryprotected topological phases. For example, a quadratic Lindbladian respecting only PHS represents a dissipative topological superconductor, which will still be protected by fermion parity symmetry (as well as the Hermiticitypreserving nature of the Lindbladian) when solvability is broken. We also expect that topological features of the spectrum and steady state will remain decoupled in this limit: Unlike the Lindblad spectrum, the ground state of a closed system is not smoothly connected to the steady state of an open system with vanishingly small dissipation. Thus any topological properties of the former are not necessarily preserved in the latter.

We note in passing that the ten Lindblad symmetry classes uncovered in this Letter may have interesting implications for the spectral statistics of random dissipative systems [44-46]. Imposing symmetries on the Lindbladian may result in universal features of the complex spectrum, in analogy with the Altland-Zirnbauer random matrix classification of Hamiltonian dynamics.

In summary, we have discovered a topological classification which constrains the dynamics of open femionic systems described by a Lindblad master equation. Specifically, we have demonstrated that the addition of symmetry-preserving dissipators will ensure that edge modes of the Lindbladian have phase oscillations which are pinned to lie in the frequency gap, but will generically acquire a nonzero lifetime. This causes the topological properties of the spectrum to decouple from those of the steady state. Our work provides a framework to systematically understand the protection of topological edge modes in the presence of both dissipation and internal dynamics.
M. M. thanks Jan Carl Budich for helpful discussions. This work was supported by an EPSRC studentship, ESPRC Grant No. EP/P034616/1, and by a Simons Investigator Award.

[1] M. Z. Hasan and C. L. Kane, Rev. Mod. Phys. 82, 3045 (2010).

[2] X.-L. Qi and S.-C. Zhang, Rev. Mod. Phys. 83, 1057 (2011).

[3] K. von Klitzing, Rev. Mod. Phys. 58, 519 (1986).

[4] J. Alicea, Rep. Prog. Phys. 75, 076501 (2012).

[5] M. S. Rudner, N. H. Lindner, E. Berg, and M. Levin, Phys. Rev. X 3, 031005 (2013).

[6] B. Galilo, D. K. K. Lee, and R. Barnett, Phys. Rev. Lett. 115, 245302 (2015).

[7] D. V. Else and C. Nayak, Phys. Rev. B 93, 201103(R) (2016).

[8] C. W. von Keyserlingk and S. L. Sondhi, Phys. Rev. B 93, 245145 (2016).

[9] A. C. Potter, T. Morimoto, and A. Vishwanath, Phys. Rev. X 6, 041001 (2016).

[10] R. Roy and F. Harper, Phys. Rev. B 96, 155118 (2017).

[11] M. McGinley and N. R. Cooper, Phys. Rev. Lett. 121, 090401 (2018).

[12] M. McGinley and N. R. Cooper, Phys. Rev. B 99, 075148 (2019).

[13] S. Lieu, Phys. Rev. B 98, 115135 (2018).

[14] K. Kawabata, K. Shiozaki, M. Ueda, and M. Sato, Phys. Rev. X 9, 041015 (2019).

[15] H. Zhou and J. Y. Lee, Phys. Rev. B 99, 235112 (2019).

[16] C.-H. Liu and S. Chen, Phys. Rev. B 100, 144106 (2019).

[17] T. Prosen, New J. Phys. 10, 043026 (2008).

[18] T. Prosen, J. Stat. Mech. (2010) P07020.

[19] D. Bernard and A. LeClair, A classification of nonHermitian random matrices, in Statistical Field Theories, edited by A. Cappelli and G. Mussardo (Springer Netherlands, Dordrecht, 2002), pp. 207-214.

[20] S. Diehl, E. Rico, M. A. Baranov, and P. Zoller, Nat. Phys. 7, 971 (2011).

[21] C.-E. Bardyn, M. A. Baranov, C. V. Kraus, E. Rico, A. İmamoğlu, P. Zoller, and S. Diehl, New J. Phys. 15, 085001 (2013).

[22] M. Goldstein, SciPost Phys. 7, 067 (2019).

[23] G. Shavit and M. Goldstein, arXiv:1903.05336.

[24] O. Viyuela, A. Rivas, and M. A. Martin-Delgado, Phys. Rev. Lett. 112, 130401 (2014).

[25] J. C. Budich and S. Diehl, Phys. Rev. B 91, 165140 (2015).

[26] C.-E. Bardyn, L. Wawer, A. Altland, M. Fleischhauer, and S. Diehl, Phys. Rev. X 8, 011035 (2018).

[27] G. Lindblad, Commun. Math. Phys. 48, 119 (1976).

[28] M. van Caspel, S. E. T. Arze, and I. P. Castillo, SciPost Phys. 6, 26 (2019).

[29] A. Altland and M. R. Zirnbauer, Phys. Rev. B 55, 1142 (1997).

[30] A. Kitaev, AIP Conf. Proc. 1134, 22 (2009).

[31] S. Ryu, A. P. Schnyder, A. Furusaki, and A. W. W. Ludwig, New J. Phys. 12, 065010 (2010). 
[32] See Supplemental Material at http://link.aps.org/supplemental/ 10.1103/PhysRevLett.124.040401 for numerical results on the dissipative SSH chain, a discussion of the conditions to satisfy the non-Hermitian time-reversal symmetry Eq. (6), and a proof that spectral and steady-state properties of the Lindbladian are independent, which contains Refs. [33-36].

[33] H. Breuer and F. Petruccione, The Theory of Open Quantum Systems (Oxford University Press, Oxford, 2002).

[34] C.-K. Chiu, J. C. Y. Teo, A. P. Schnyder, and S. Ryu, Rev. Mod. Phys. 88, 035005 (2016).

[35] A. Kitaev, Ann. Phys. (Amsterdam) 321, 2 (2006).

[36] W. P. Su, J. R. Schrieffer, and A. J. Heeger, Phys. Rev. Lett. 42, 1698 (1979).

[37] F. Dangel, M. Wagner, H. Cartarius, J. Main, and G. Wunner, Phys. Rev. A 98, 013628 (2018).

[38] A. Y. Kitaev, Phys. Usp. 44, 131 (2001).
[39] K. Esaki, M. Sato, K. Hasebe, and M. Kohmoto, Phys. Rev. B 84, 205128 (2011).

[40] J. C. Budich, S. Walter, and B. Trauzettel, Phys. Rev. B 85, 121405(R) (2012).

[41] A. Carmele, M. Heyl, C. Kraus, and M. Dalmonte, Phys. Rev. B 92, 195107 (2015).

[42] L. Campos Venuti and P. Zanardi, Phys. Rev. A 93, 032101 (2016).

[43] V. V. Albert, B. Bradlyn, M. Fraas, and L. Jiang, Phys. Rev. X 6, 041031 (2016).

[44] T. Can, V. Oganesyan, D. Orgad, and S. Gopalakrishnan, Phys. Rev. Lett. 123, 234103 (2019).

[45] S. Denisov, T. Laptyeva, W. Tarnowski, D. Chruściński, and K. Życzkowski, Phys. Rev. Lett. 123, 140403 (2019).

[46] L. Sá, P. Ribeiro, and T. Prosen, arXiv:1905.02155. 